\title{
Factors associated with oral health: a multivariate analysis of results from the 1998 Adult Dental Health survey
}

\author{
E. Treasure, ' M. Kelly, ${ }^{2}$ N. Nuttall,, ${ }^{3}$ J. Nunn, ${ }^{4}$ G. Bradnock, ${ }^{5}$ and D. White, ${ }^{6}$
}

\begin{abstract}
This paper presents results from the 1998 Adult Dental Health Survey using multivariate analysis. This enables analysis of several variables at one time to see which are having an effect on people's oral health. The paper compares these variables to a variety of outcome measures and makes recommendations for dental practice.
\end{abstract}

$\mathrm{T}$ he national surveys of Adult Dental Health have given a 10-yearly summary of the clinical condition of adults in the United Kingdom on three previous occasions. The fourth report in the series was published in March of 2000. For the 1998 survey 4,984 addresses were identified at which all adults over 16 in residence were asked to take part in the survey; $21 \%$ of households refused and no contact was made at $5 \%$ of them. In total, 6,204 adults were interviewed following which those with some teeth were asked to undergo a dental examination; $3,817(72 \%)$ of those eligible agreed. A weighting system based on some of the interview responses of those who consented to be dentally examined and those who were interviewed but not dentally examined was used to reduce bias from non-response. ${ }^{1}$ The survey was carried out under the auspices of the Office of National Statistics together with the Universities of Birmingham, Dundee, Newcastle-upon-Tyne and Wales.

The national surveys of adult dental health have tended to look at factors associated with oral health as single factors. For example the level of total tooth loss has been shown to be related to social class and to the part of the country people live in; but this raises the issue of whether differences in

${ }^{1}$ Professor, University of Wales, Cardiff; ${ }^{2}$ Senior Social Survey Officer, Office for National Statistics, London;

${ }^{3}$ Senior Research Fellow, Dental Health Services

Research Unit, University of Dundee; ${ }^{4}$ Senior

Lecturer, University of Newcastle upon Tyne; ${ }^{5}$ Senior

Lecturer, ${ }^{6}$ Lecturer, University of Birmingham

${ }^{*}$ Correspondence to: Professor E T Treasure, The

Dental School, Heath Park, Cardiff CF14 4XY

email:treasureet@cardiff.ac.uk

REFEREED PAPER

Received 13.06.00; Accepted 31.07.00

(C) British Dental Journal 2001; 190: 60-68

\section{In brief}

- Variations in disease are caused by more than social class structure. It is likely that other complex sociobehavioural factors are at work.

- The survey supports the use of fissure sealants and encouraging people to brush their teeth more frequently.

- There is a huge difference in the experience of restorative dentistry in the average 20 -year-old compared with the average 30 -year-old.

total tooth loss patterns between countries is simply because they have different social class structures. In statistics there are a group of tests (multivariate models) designed specifically to select out which socio-demographic or behavioural factors were associated with clinically measured aspects of oral health. The data on the clinical measures of oral health were analysed using age standardisation and two multivariate modelling techniques; multiple regression for continuous dependent variables (eg number of teeth) and logistic regression for dependent variables with two outcomes (eg had artificial crowns or did not have artificial crowns).

The factors used in the modelling were:

Socio-Demographic:

- Age

- Gender

- Social class of head of household

- Educational attainment

- English region (North, South, Midlands) and country (Scotland, Northern Ireland Wales)
- Marital status

- Economic status.

Behavioural

- Usual reason for dental attendance

- Frequency of tooth cleaning

- Use of additional methods for cleaning teeth (eg floss).

In the figures 'triangles' are used to indicate where a variable was entered into the model but found to have no effect. In the analysis used these variables were then dropped.

\section{What characteristics are associated with total tooth loss?}

Table 1 shows the likelihood of a person with a given characteristic (eg a person from Scotland) having no natural teeth. The 'odds ratio' is simply the number of times more likely it is that a particular type of person will have no teeth in comparison to another. The 'another' person in this table is the one whose odds are 1.00. For example, for people of different ages those aged over 75 were 144 times more likely to have lost all their teeth in comparison with 16-44 year olds. Once all other factors in the model were taken into account educational attainment had the next largest effect after age on the odds of being edentate. The odds of being edentate were almost nine times higher for those adults with no qualification and four times higher for those with qualifications below degree level. Being from the north of Great Britain was also a factor that had an effect with the odds of having no teeth rising as distance from the South of England increased. The effect was not however observable in Northern Ireland. Gender was not found to be a factor that altered the odds of a person being edentate and this differs from the bivariate analysis.

\section{What characteristics were associated with the number of natural teeth in the mouth? \\ Multiple regression modelling was carried out to discover which combinations of}


socio-demographic and behavioural factors independently affected the number of teeth among dentate adults (Fig. 1). The coefficients can be used to estimate the average number of teeth a person with given characteristics would have by adding them up for a person and adding this to a constant calculated by the model. For example, a 32-year-old married women who has a university degree and comes from a non-manual working background living in Scotland and who brushes her teeth twice a day and claims to attend for regular check-ups would be expected to have 28.44 teeth.

\section{Constant}

22.40

Age 25-34

Woman

Scotland

$-0.85$

Non-manual working background $\quad+0.64$

Degree

$+0.99$

Married

$+0.40$

Tooth cleaning twice daily $\quad+0.54$

Regular check-ups $\quad+0.54$

Total 28.44

Whether a person used an additional method of tooth cleaning was entered into the model but was not found to have any independent effect on the number of teeth among dentate adults.

It is often pointed out that the dental diseases are a largely preventable. So why, if this is the case, are people who live in Scotland likely to have 1.82 fewer teeth than someone from the South of England once all other factors are taken into account? Is there a factor simply in being from Scotland that could account for an almost two tooth disadvantage over the South of England? The answer is that other factors will be at work as well, which is indicated by the $\mathrm{R}^{2}$ value that shows how much of the variation is explained by the model, in this case $44 \%$. There are some obvious candidates that might add to the explanatory power of these models one of which would be dietary factors such as eating sugary foods or drinks.

\section{What can this sort of analysis tell us about the experience of dental disease among young adults?}

Adults under the age of 35 were found to have a relatively low experience of dental disease and treatment. By concentrating on this group it was planned to exclude the historical burden of disease and concentrate on the factors associated with the number of sound and untreated teeth.

This analysis again indicated that age was the most important factor followed by region or country. However in the case of young adults it was the Midlands where

Table I Likelihood of an adult having lost all their natural teeth (based on odds ratios from logistic regression)

Dentate adults

Characteristic

$16-44$

45-54

$55-64$

65-74

75 and over

English region or country

North

Midlands

United Kingdom

South

Wales

Scotland

Northern Ireland

Social class of head of household

$\begin{array}{ll}\text { I, II, IIINM } & 1.00 \\ \text { IIIM } & 2.14^{\ddagger} \\ \text { IV, V } & 2.21^{\ddagger}\end{array}$

Educational attainment

At degree level or above

Below degree level

No qualifications

1.00

$3.95^{\ddagger}$

$8.79^{\ddagger}$

Marital status

Single

$1.45^{*}$

Married/cohabiting

1.00

Divorced/separated

Widowed

1.43

$\mathrm{R}^{2}$

$1.62^{\ddagger}$

0.50
*Significant at $95 \%$ level
†Significant at $99 \%$ level
${ }_{\text {†Significant at } 99.9 \% \text { level }}$

people had most sound and untreated teeth on average and Northern Ireland where people had fewest. A potentially important finding was that those adults who had some fissure sealants had 1.32 more sound and untreated teeth than those who did not have sealants. This suggests that, at a population level, sealants play an effective role in preventing disease at this age. Only $24 \%$ of the variation was explained by the factors included in this model (Fig. 2).

\section{What factors are associated with having dental caries or unsound teeth?}

Because of the huge proportion of subjects with no decayed or unsound teeth and that the remaining subjects had very few decayed teeth this oral health measure was treated as a dichotomous variable. This measure showed very different associations in comparison with the other clinical measurements. Firstly, there was a different relationship with age. People aged 25 to 34 and those aged 45 to 54 years were the only ones to have significantly greater odds of having a decayed or unsound tooth. Living in the north of Great Britain was again associated with poorer dental health: those in the north of England were 1.86 times more likely to have some untreated decay than those living in the south of England. Only one of the behavioural variables varied significantly between groups and that was, 


\section{adult dental health survey}

Fig. I Factors independently affecting the

number of teeth based on coefficients from

multiple regression

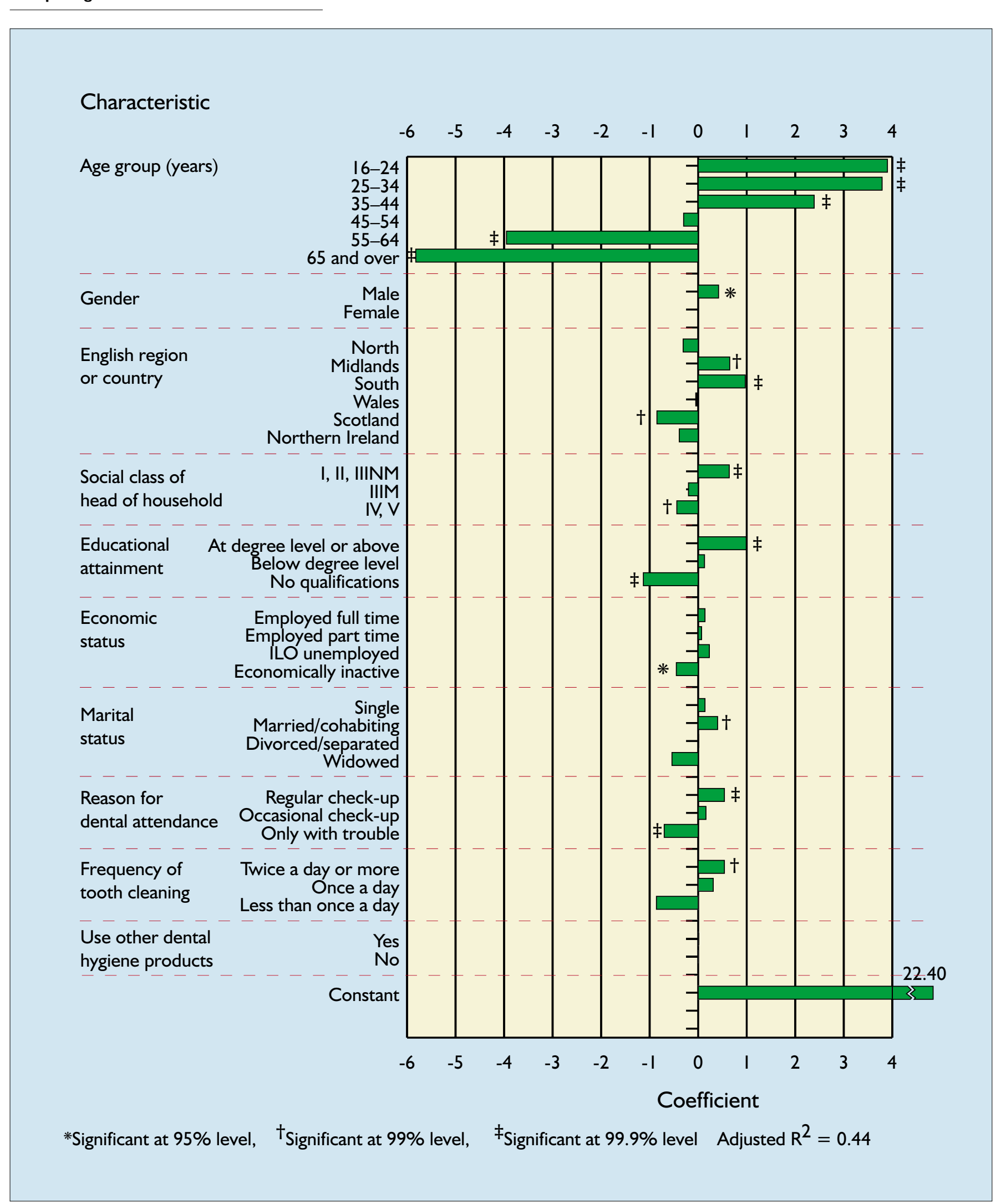




\section{Characteristic}

$\begin{array}{llllllllllll}-3.0 & -2.5 & -2.0 & -1.5 & -1.0 & -0.5 & 0.0 & 0.5 & 1.0 & 1.5 & 2.0 & 2.5\end{array}$

Age group (years)

$16-19$
$20-24$
$25-29$

$25-29$

Gender

30-34

Male

English region

or country

Female

North

Midlands

South

Wales

Scotland

Northern Ireland

$\begin{array}{ll}\text { Social class of } & \text { I, II, IIINM } \\ \text { head of household } & \text { IIIM } \\ \text { IV, V }\end{array}$

Educational At degree level or above attainment

Below degree level

No qualifications

Economic

status

Employed full time

Employed part time

ILO unemployed

Economically inactive

Marital

status

Single

Married/cohabiting

Divorced/separated

Reason for

dental attendance

Frequency of

tooth cleaning

Regular check-up

Occasional check-up

Only with trouble

Twice a day or more

Once a day

Less than once a day

Use other dental

hygiene products

Has fissure

sealants

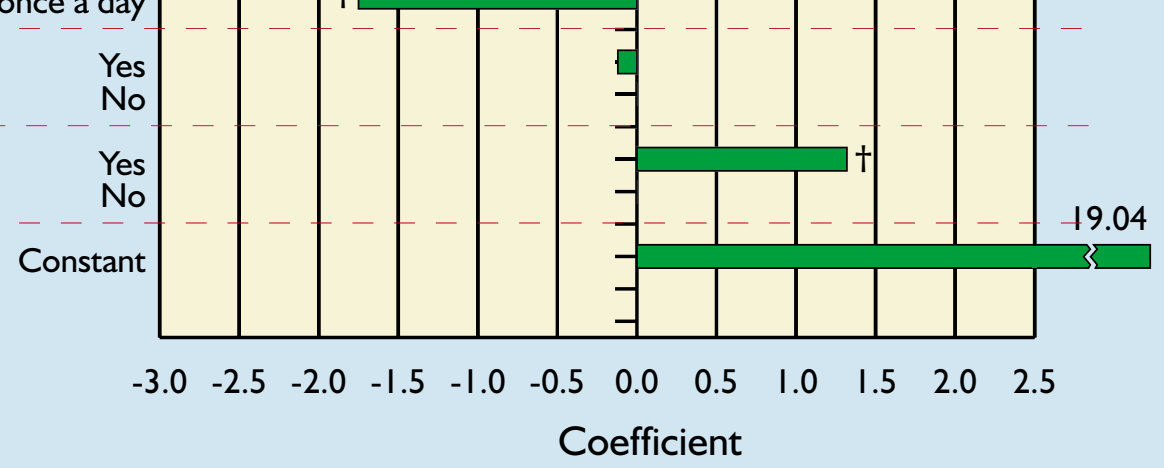

*Significant at $95 \%$ level, $\quad{ }^{\dagger}$ Significant at $99 \%$ level, $\quad{ }^{\ddagger}$ Significant at $99.9 \%$ level $\quad$ Adjusted $R^{2}=0.24$ 


\section{Characteristic}

\section{$\begin{array}{lllllllll}0.0 & 0.5 & \text { I.0 } & \text { I.5 } & 2.0 & 2.5 & 3.0 & 3.5 & 4.0\end{array}$}

Age group (years)

16-24

25-34

$35-44$

$45-54$

$55-64$

65 and over

Gender

Male

English region

or country
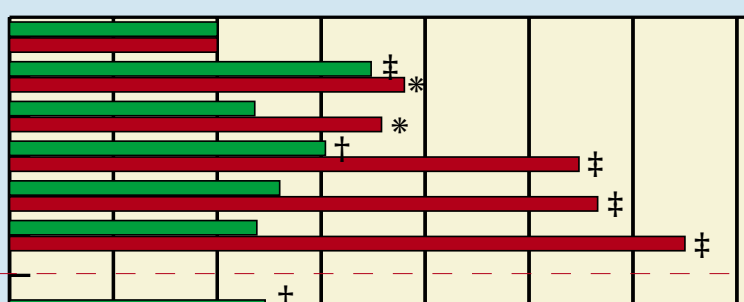

Female

North

Midlands

South

Wales

Scotland Northern Ireland

Social class of

head of household

I, II, IIINM
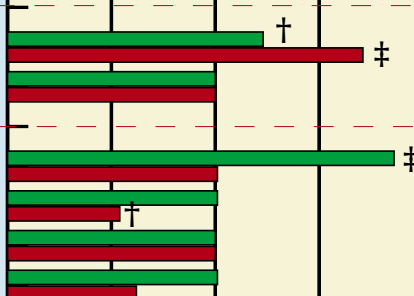

$\frac{1}{2-1}$
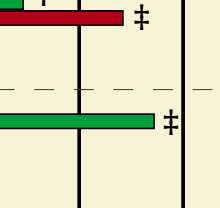
Educational

At degree level or above

Below degree level

No qualifications

Marital

Single

status

Married/cohabiting

Divorced/separated

Widowed

Reason for

dental attendance

Regular check-up

Occasional check-up

Only with trouble

Use other dental

hygiene products

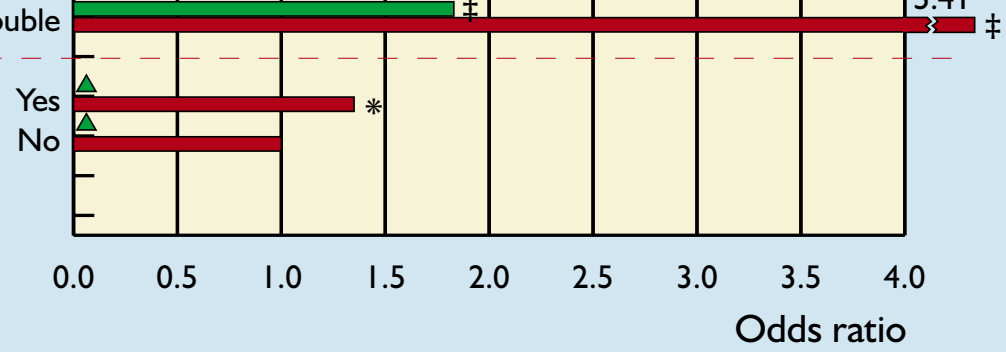

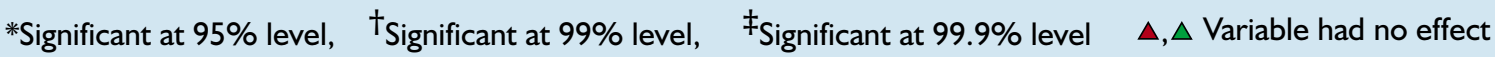
Adjusted $\mathrm{R}^{2}=0.08$ (green) and 0.19 (red) 
Fig. 4 Likelihood of a dentate adult having

12 or more restored (otherwise sound) teeth

or having artificial crowns (based on odds

ratios from logistic regression)

Odds ratio of having 12 or more restored (otherwise sound) teeth

Odds ratio of having one or more artificial crown

Characteristic

Age group (years)

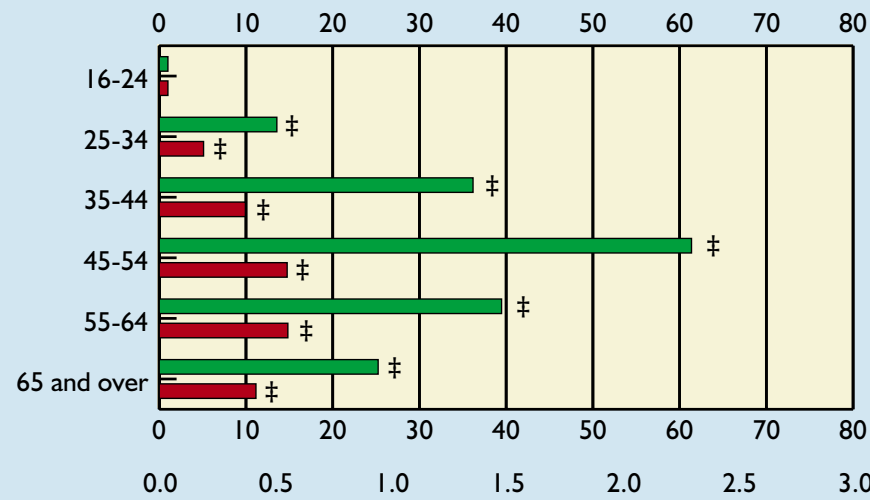

Gender

English region

or country

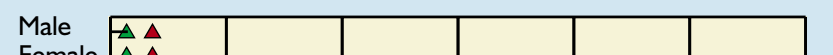

Female $\triangle \Delta$

North

Midlands $\mathbf{A}$

Wales

Scotland

Northern Ireland

Social class of

head of household

I, II, IIINM

IIIM

IV,

Educational At degree level or above

attainment

Now degree level

No qualification

Economic

status

Employed full time

Employed part time

ILO unemployed Economically inactive

Marital

Single

status

Married/cohabiting

Divorced/separate

Widowed

Reason for

Regular check-up

dental attendance

Occasional check-up

Only with trouble

Frequency of

tooth cleaning

More than twice a day

Twice a day

Once a day

Less than once a day

Use other dental

hygiene products

Yes
No

No

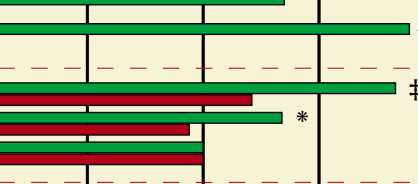


those who reported attending the dentist only with trouble had significantly higher odds of having a decayed or unsound tooth. Factors that were not found to be significant by the model were whether people were in employment or not, frequency of tooth cleaning and the use of additional dental hygiene products along with toothbrush or toothpaste. It is clear that as only $8 \%$ of the model was explained there are many factors influencing this measure that are not included in this analysis (Fig. 3).

The picture for unrestorable decay was different. The factor that had the strongest association with having unrestorable caries was the reported attendance pattern at a dentist. People who said they attended only when they had trouble had odds five times higher than those who said they went to the dentist for a regular check-up. The other strong relationship was with age where the odds increased with age so that at age 65 the odds were over 3 times greater than at age 16 to 24. People in the Midlands had significantly lower odds of having an unrestorable tooth in comparison with the other regions and countries of the United Kingdom. Women were also less likely to have some unrestorable caries in comparison with men. Marital status, economic status or reported frequency of tooth cleaning were not significant factors. The model explained $19 \%$ of the variation.

\section{What factors are associated with the experience of restorative treatment in adults?}

Two measures were used to try to gain some understanding of the factors underlying adults who have had some restorative treatment. These were whether a person had 12 restored but otherwise sound teeth or if they had an artificial crown (Fig. 4).

As might be expected age was the most important factor in determining the odds of an adult having either 12 or more restored teeth or an artificial crown.

The chance of having 12 or more filled teeth increased markedly with age until 55 ; and then dropped. A dentate person aged 50 was over 60 times more likely to have 12 or more filled teeth than someone aged 20 . However, more remarkable was the huge difference just between the two youngest age groups; the average 30-year-old was 13 times more likely to have 12 or more filled teeth than the average adult aged 20. This was a much more marked difference than between any other two consecutive age groups; 40-year-olds were only about 3 times more likely to have 12 or more filled teeth than 30-year-olds. This illustrates something that comes out in other analyses in the survey report which is that a new generation is entering the adult population who have much less experience of restorative treatment.

It should come as no surprise that dental attendance behaviour was a strong factor in the experience of having restorative treatment. Those adults who said they usually went to a dentist for regular check-ups were 2.74 times more likely to have 12 or more sound and restored teeth and 1.66 times more likely to have an artificial crown than those who said they only went to a dentist when having some dental trouble.

\section{What factors are associated with periodontal conditions?}

In the logistical regression for periodontal condition the measure used was loss of attachment of $4 \mathrm{~mm}$ or more (Fig. 5). Age was the factor most strongly related to periodontal conditions; adults over 65-yearsold were 33 times more likely to have a loss of attachment of $4 \mathrm{~mm}$ or more in comparison with those aged 16 to 24 . Men had significantly higher odds of having this degree of loss of attachment as did those with education below degree level. It is interesting that none of the behavioural factors that were examined were found to be significant in this model. Twenty-eight per cent of the variation is explained by this model.

\section{Implications for the practice}

This multivariate analysis gives some indication of the factors that may be responsible for variations in oral health. Living in Scotland or the north of England increases your chance of having lost all your teeth, or if you have some teeth, having some with dental caries. Furthermore, this association is not only caused by social class structure of the regions and countries of the United King- dom which suggests that other more complex socio-behavioural factors are at work. Thus in order to bring about improvements in this area it is necessary to understand what these are. Findings such as these have also been described in other areas of healthcare. ${ }^{2}$

Trying to understand the reasons behind this variation can only be supposition in many instances but there are some interesting variations that may be worth looking at. For example why is Northern Ireland not a region associated with high levels of edentulousness or in having low numbers of teeth but does have significantly fewer sound and untreated teeth? Does this suggest a very different pattern of extraction in Northern Ireland more similar to the South of England although in other ways the disease pattern would appear to be very different from the south?

There are some tantalising suggestions in these data which it is not possible to substantiate. For example, is it only coincidence that the Midlands have the second highest coefficient for number of teeth and the greatest coefficient for the numbers of sound and untreated teeth? However this improved situation does not carry through to current decay or in the number of restorations. It is tempting to suggest that fluoride might be responsible for the greater chances of having more teeth and more healthy teeth but why not the other factors? It is not possible to say but it gives pointers for further research.

Why is there not an age relationship in the younger groups for unsound and untreated teeth? This suggests that the odds of having a decayed tooth are similar in these younger age groups. Does this mean that there is a problem accessing services or that as fast as these teeth are treated fresh decay is arising?

The findings regarding the effect of going to the dentist are quite difficult to interpret. Those who say they visit a dentist for regular check-ups have greater odds of having less decayed teeth, less unrestorable teeth but more filled teeth, more chance of having a crown and less sound and untreated teeth. It is all too easy to decide that these factors are caused by over-treatment but it is just not possible to deduce that from these data. This is because nothing is known about the 
Fig. 5 Likelihood of a dentate adult having periodontal loss of attachment of $4 \mathrm{~mm}$ or more (based on odds ratio from logistic

regression)

Characteristic

Age group (years)

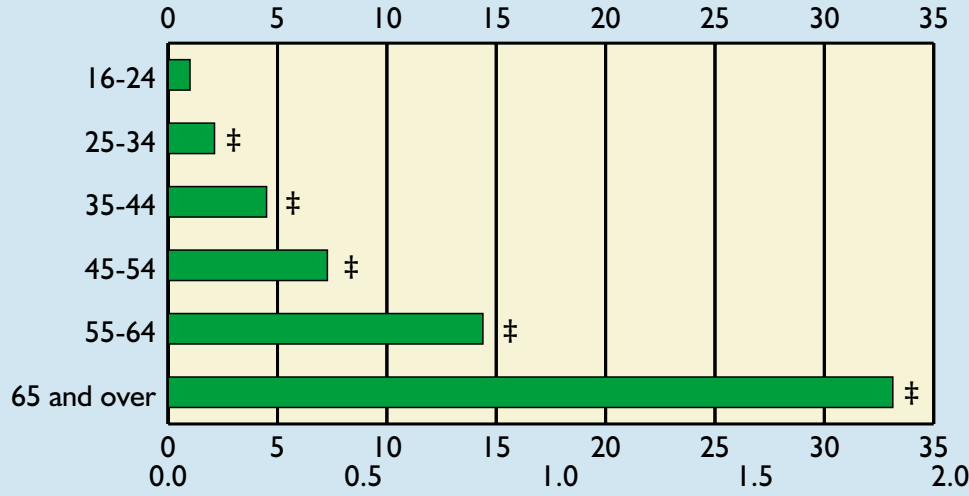

Gender

English region or country

$$
\text { Male }
$$

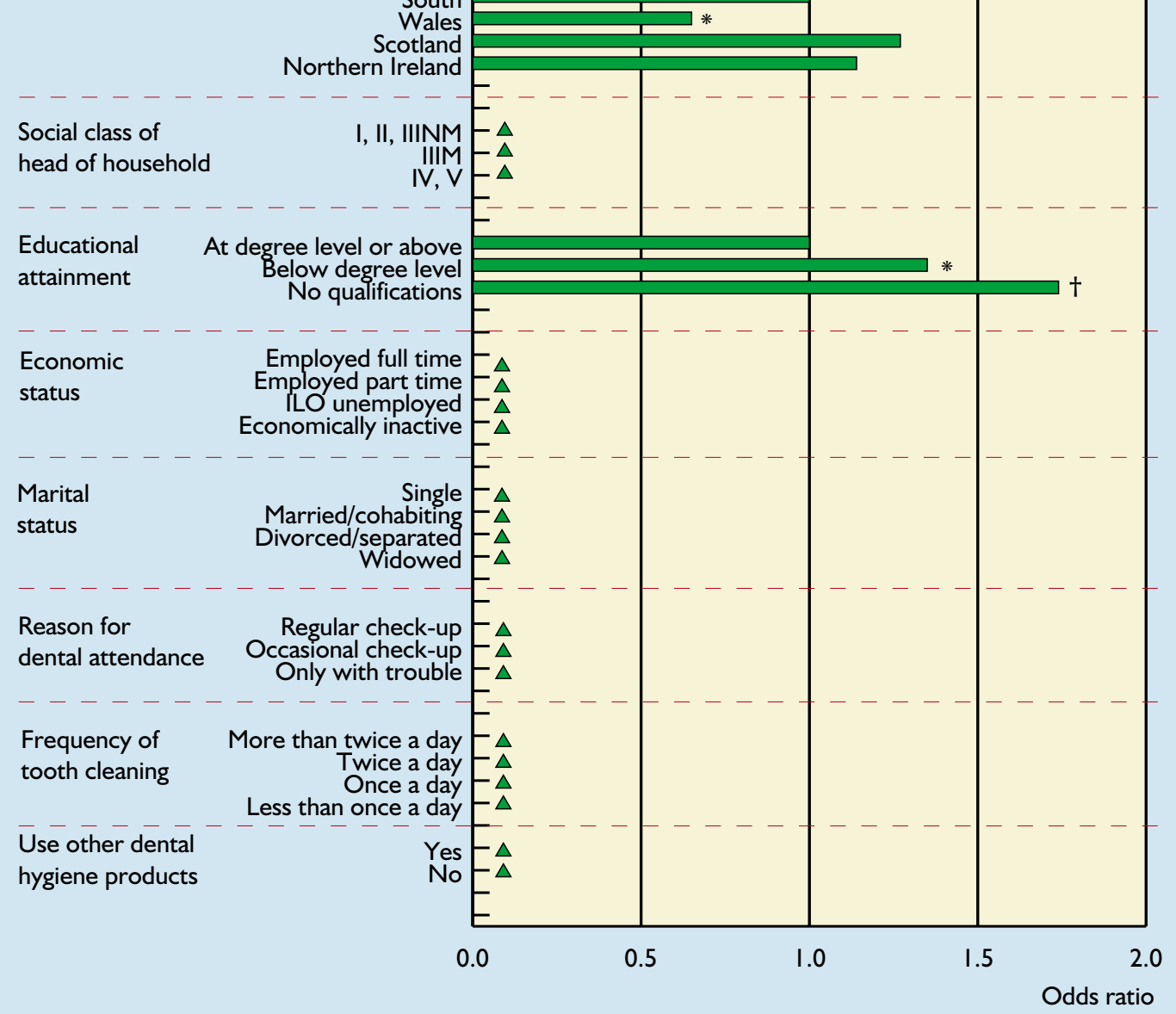

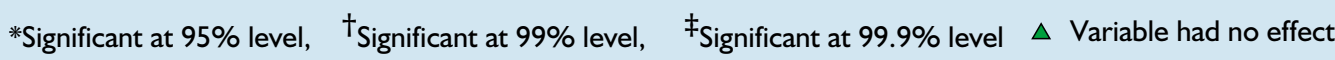

$$
\text { Adjusted } \mathrm{R}^{2}=0.28
$$


incidence (as opposed to prevalence) of dental disease in this population and its subgroups. What has not been investigated here is the odds of having a functioning dentition related to reported attendance pattern. This is worthy of further investigation.

The findings relating to fissure sealants may be important in that they suggest that people who have fissure sealants in place are likely to have more sound and untreated teeth than others. However it is not possible to comment on the overall effectiveness of fissure sealing teeth as we do not know which other subjects had received sealants and then lost them or who had them replaced with a restoration. Similarly toothbrushing was positively associated with the number of sound and unrestored teeth.

Periodontal loss of attachment was not affected by any of the behavioural factors included in the models. It is unfortunate that smoking was omitted from the questionnaire and so could not be included. This does cause problems in how we should explain this disease to patients as there are no behavioural factors described here that can be recommended as making a real difference.

Being older was not associated with having a greater risk of having a decayed tooth but the oldest groups in this study did have a greater risk of having an unrestorable tooth. With the increasing proportion of the population who are ageing this must be of concern to the practitioner. What is to be done to reduce this more complex treatment need in the very oldest in society? Taken together it could be suggested that there is a need for a greater emphasis for a more preventive approach to dental practice. In particular the survey supports the use of fissure sealants and encouraging people to brush their teeth more frequently.
This article has been refereed under the British Dental Journal reviewing process. Full details of sample numbers and the criteria for the clinical examinations can be found in the survey report. We would like to acknowledge the work of Alison Walker and other staff of the Office for National Statistics.

This work was undertaken by a consortium comprising the Office for National Statistics and the Dental Schools of the Universities of Birmingham,

Dundee, Newcastle and Wales who received funding from the United Kingdom Health Departments; the views expressed in this publication are those of the authors and not necessarily those of the Health Departments nor of the other members of the consortium.

1 Kelly M, Steele J, Nuttall N, Bradnock G, Morris J, Nunn J, Pine C, Pitts N, Treasure E, White D. Adult Dental Health Survey — Oral Health in the United Kingdom 1998. London: The Stationery Office, 2000.

2 Acheson D. Independent Inquiry into Inequalities in Health. The Stationery Office, ISBN $0113221738,1998$. 